\title{
Extracorporeal Shock Wave Treatment (ESWT) enhances the in vitro-induced differentiation of human tendon-derived stem/ progenitor cells (hTSPCs)
}

\author{
Laura Leone ${ }^{1}$, Salvatore Raffa ${ }^{1,4}$, Mario Vetrano², Danilo Ranieri ${ }^{1}$, Florence Malisan ${ }^{3}$, \\ Cristina Scrofani ${ }^{1}$, Maria Chiara Vulpiani ${ }^{2}$, Andrea Ferretti ${ }^{2}$, Maria Rosaria Torrisi ${ }^{1,4}$ \\ and Vincenzo Visco ${ }^{1,4}$ \\ ${ }^{1}$ Department of Clinical and Molecular Medicine, Faculty of Medicine and Psychology, "Sapienza" University of Rome, Rome, \\ Italy \\ 2 Department of Orthopedics and Traumatology, Sant'Andrea Hospital, Faculty of Medicine and Psychology, "Sapienza" \\ University of Rome, Rome, Italy \\ 3 Department of Biomedicine and Prevention, Laboratory of Signal Transduction, "Tor Vergata" University of Rome, Rome, \\ Italy \\ ${ }^{4}$ Cellular Diagnostics Unit, Sant'Andrea Hospital, Rome, Italy \\ Correspondence to: Vincenzo Visco, email: vincenzo.viscol@uniromal.it \\ Keywords: differentiation, tendon, stem cells, healing, ESWT, Pathology Section \\ Received: August 03, 2015 \\ Accepted: January 13, 2016 \\ Published: January 28, 2016
}

\section{ABSTRACT}

Extracorporeal shock wave therapy (ESWT) is a non-invasive and innovative technology for the management of specific tendinopathies. In order to elucidate the ESWT-mediated clinical benefits, human Tendon-derived Stem/Progenitor cells (hTSPCs) explanted from 5 healthy semitendinosus (ST) and 5 ruptured Achilles (AT) tendons were established. While hTSPCs from the two groups showed similar proliferation rates and stem cell surface marker profiles, we found that the clonogenic potential was maintained only in cells derived from healthy donors. Interestingly, ESWT significantly accelerated hTSPCs differentiation, suggesting that the clinical benefits of ESWT may be ascribed to increased efficiency of tendon repair after injury.

\section{INTRODUCTION}

The main cellular component of the human tendon consists of tenocytes that represent only the $5 \%$ of the normal tissue volume and are responsible for its maintenance and repair [1]. However, histological findings of injured tendons showed the presence of cells characterized by several phenotypes, suggesting that a possible multilineage differentiation potential may be involved either in tissue repair or in pathophysiology of such diseases, frequently characterized by fatty degeneration and chondroid calcification $[2,3]$. On the other hand, the recovery of a normal function of injured tendons represents an ambitious goal in this field and various cell-based therapies with regenerative purpose for tendon-related pathologies have been recently performed [4-6]. The replacement of injured tenocytes and the restoration of a normal tissue homeostasis have been efficiently obtained by the injection of mesenchymal stem cells (MSCs) in the area of damaged tendons [5, 7].
Pathophysiology of tendinopathy, a degenerative disorder of the tendons, is still unclear and a better understanding of the cellular mechanisms involved in the healing process occurring in this pathology could strongly improve its therapeutic management [2]. The tendon healing is a multistep process involving inflammatory cells, cytokine release, extracellular matrix (ECM) remodelling and neoangiogenesis. In fact, tendinopathies can also be treated with autologous Platelet-rich plasma (PRP), containing large amounts of growth factors, some of them favoring tendon healing by cell recruitment and differentiation, while its clinical effectiveness remains matter of debate [8-10].

ESWT is an alternative treatment of some chronic tendinopathies. In order to explain its clinical benefits, we recently demonstrated that ESWT enhances cell proliferation, migration and secretory activity of human primary cultured tenocytes [11-13]. Previous reports suggested that the physical stimulation provided by shock waves could involve recruitment and further differentiation of stem cells [14-17]. 
Intriguingly, human Tendon-derived Stem/ Progenitor cells (hTSPCs) were efficiently isolated from tendons of various species, including human hamstring tendons [18-20]. These local progenitor cells showed a self-renewal capacity, the potential to differentiate along an assortment of mature cell lineages -including tenocytes [21]- and are supposed to enhance tendon repair in several models [22-24]. Therefore, it has been recently proposed a function of resident stem cells in promoting tendon repair [1].

In light of those observations, we aimed to investigate the possible effects of shock wave exposure on hTSPCs explanted from healthy semitendinosus or ruptured Achilles tendons. In order to assess the ESWT putative burden on cell differentiation, we treated our cultures at the optimal dose accurately selected in previous investigations [11-13]. Our data show that this treatment significantly promotes the in vitro-induced hTSPCs differentiation.

\section{RESULTS}

\section{ST and AT cells share similar characteristics}

We established 10 different primary cultures of human tenocytes, 5 derived from healthy semitendinosus (ST) and 5 from ruptured Achilles (AT) tendons (Table 1), as previously described [12]. Human tendon-derived cells, both from ST or AT, were plastic-adherent and after 2 weeks of culture were subjected to cell characterization.

In both ST and AT cultures, phase contrast microscopy shows heterogeneous cell morphology, consisting in two main cellular patterns, as expected [1, $11,18,20,25]$. A prevalent fibroblast-like phenotype, spread on the plate and characterized by elongated cell bodies with thin processes, alternated with a minority of enlarged tenoblast-like cells (Figure 1), which become progressively more represented when cultures was reaching confluence (data not shown), as previously

\section{A}
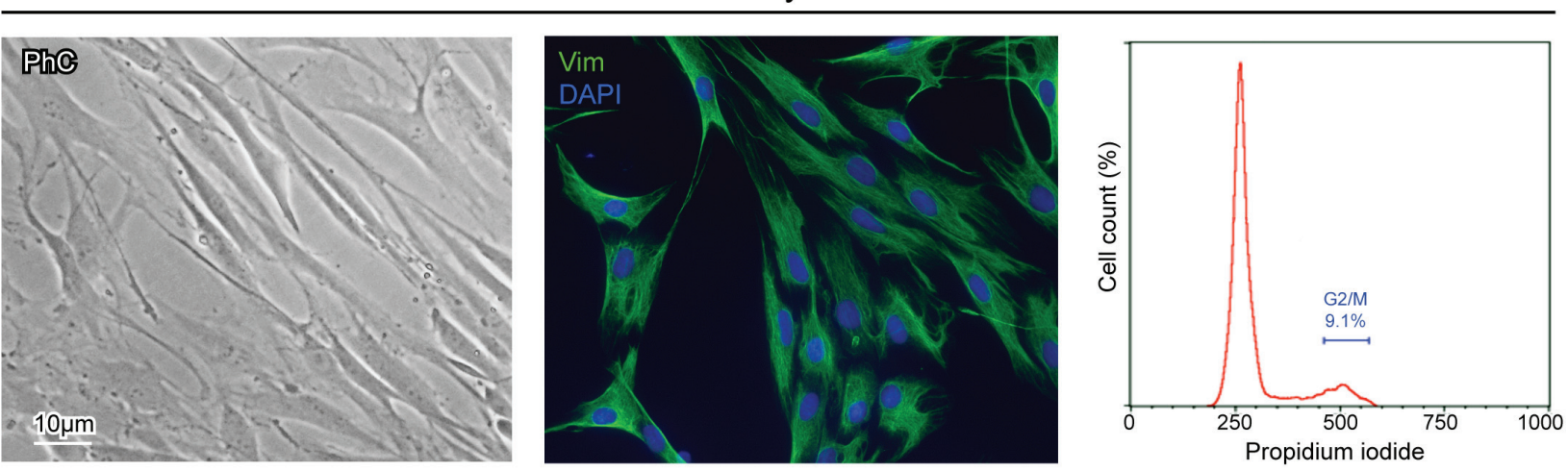

B

\section{Ruptured AT1}
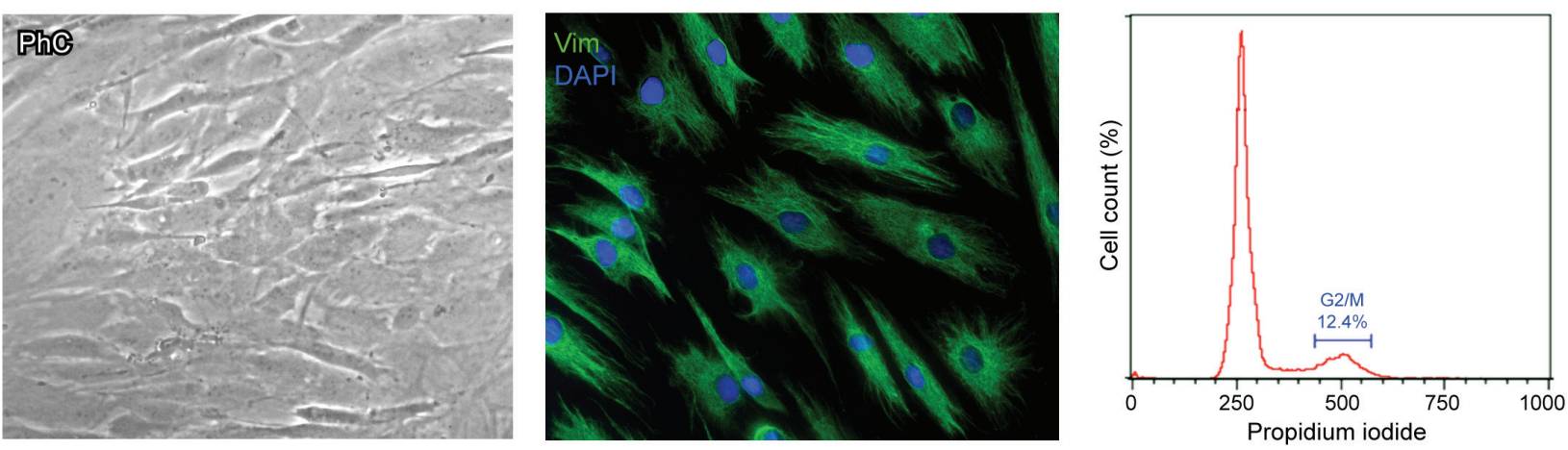

Figure 1: Culture cell characterization shows no significant differences in ST and AT cells. Morphological analysis by phase contrast microscopy, vimentin evaluation by immunofluorescence and histograms plots of cell cycle distribution by flow cytometry in ST1 A. and AT1 B. cultures, representative of healthy and ruptured human tendon-derived cells. Nuclei are stained with DAPI. Bar $10 \mu \mathrm{m}$. 
reported [11]. No significant morphological differences were detected between ST1-5 and AT1-5 cells (Table 1).

In order to evaluate possible differences in ST (ST15) and AT (AT1-5) cells, vimentin, a mesenchymal cell marker that labels cytoskeleton intermediate filaments, was then evaluated by immunofluorescence (Figure 1). A positive staining of perinuclear cytoplasmic bundles of filaments confirmed unequivocally the mesenchymal origin of all our cultures and excluded possible derangement of the cells (Table 1).

To evaluate the growth rate of ST (ST1-5) and AT (AT1-5) cells, we further analyzed the cell cycle distribution by flow cytometry (Table 1 and Figure 1). Results in Table 1 showed that both types of cultures were characterized by a similar mean percentage of cells in G2/M phase (10,34 in healthy ST1-5 and 11,86 in ruptured AT1-5), indicating the presence of proliferating cells in all our samples.

\section{Clonogenic potential is observed only on healthy human semitendinosus-derived cells}

Because stem cells typically display clonogenic potential, we tried to clone all our 10 primary cultures. We observed that they started to proliferate after few days of quiescence. Clonogenic cultures were generated by diluting suspension ( 1 cell/ $\mu \mathrm{l}$ ) (as previously described $[18,19])$ and they appeared heterogeneous in size and cell density. Therefore -according to previous authors [26]- we considered clones only those with diameter $>2 \mathrm{~mm}$. In our samples, clonogenic potential seemed to be independent on tendon-derived patient age, but exclusive of ST cells (ranging from 16 to 45 years old, see Table 1): in fact, four out of five cultures derived from healthy ST were cloned and named ST1-4/C (Figure 2A-2B), but none of the cultures from ruptured AT could be cloned (Figure 2C).


Figure 2: Cloning of tendon-derived cells. Cloning of ST1, representative of healthy ST-derived cells, one A. and two B. weeks after plating. C. Uncloned AT1, representative of cells derived from ruptured AT, 12 days after plating. Bar: $20 \mu \mathrm{m}$.


Figure 3: Expression of the differentiation marker alpha-smooth muscle actin ( $\alpha-S M A)$ in healthy ST- and ruptured AT-derived cells. A. The $\alpha$-SMA signal is present in intracellular filaments situated at the cytoplasmic periphery of all the tendon-derived cells, here represented by AT1. Nuclei are stained with DAPI. Bar $20 \mu \mathrm{m}$. B. Quantitative analysis of a-SMA-positive cells in AT, ST and $\mathrm{ST} / \mathrm{C}$ cells reveals a significant increase in ruptured AT compared to healthy ST cells (Mann-Whitney: $* p<0.05$ and $* * p<0.001 v s \mathrm{ST}$ and ST/C respectively). The data are represented with the Tukey box-and-whisker plot; the values are expressed as median \pm Interquartile Ranges (IR) from three independent experiments. 


\section{Expression of the differentiation marker alpha- smooth muscle actin ( $\alpha$-SMA) is significantly enhanced in uncloned AT cells}

Considering that several authors reported a depletion of stem cell pool and a limited differentiation potential due to aging of the donors, for this part of the study we selected 6 cultures (ST1-2, the corresponding clones ST12/C and the uncloned AT1-2) derived from the youngest donors, ranging from 17 to 25 years old [1, 25, 27, 28].

First we evaluated by immunofluorescence the expression of $\alpha$-SMA, which is a differentiation marker for activated tenocytes, as previously suggested [29], showing a signal localized in intracellular filaments, also organized in bundles, situated in the peripheral areas of the cytoplasm, (as shown in Figure 3A). Quantitative analysis of $\alpha$-SMA-positive cells revealed an increase of differentiation in uncloned AT, especially when compared to cloned ST/C, and to a lesser extent to uncloned ST cells (Figure 3B), suggesting that these different types of cultures are not at the same stage of differentiation.

A
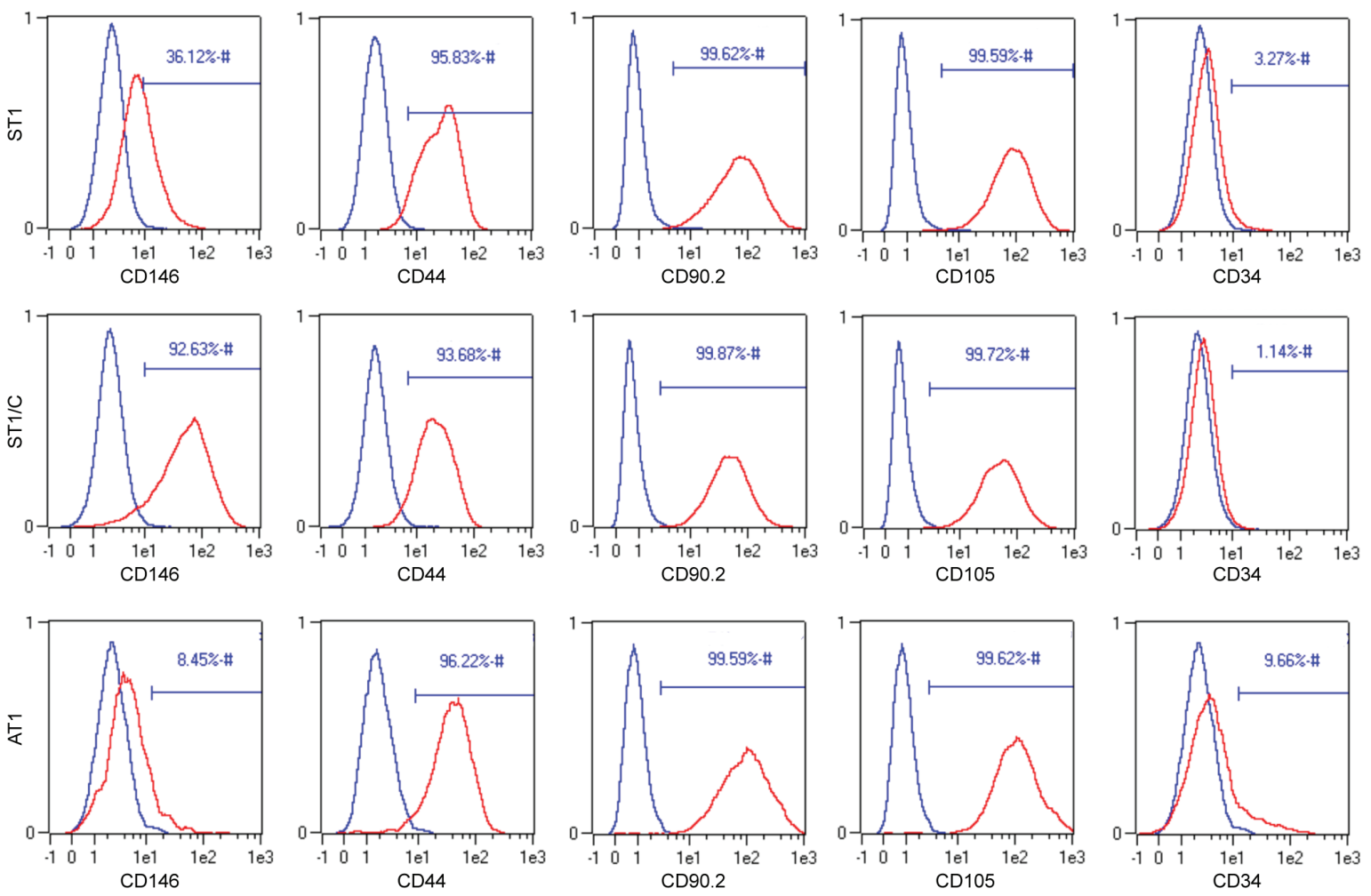

B
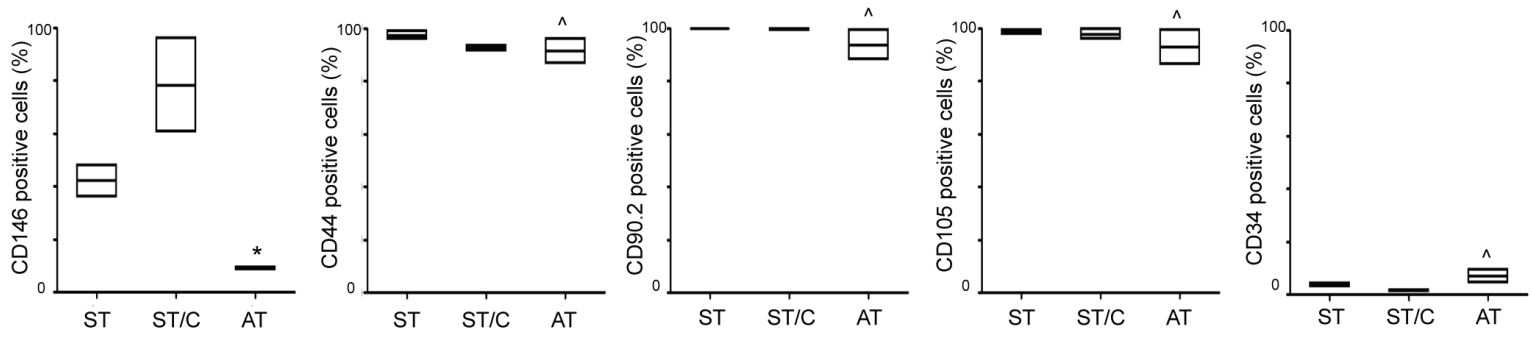

Figure 4: ST, ST/C and AT cells express typical surface stem cell markers. Typical cell surface markers are analyzed by flow cytometry in ST1-2, ST1-2/C and AT1-2 cells. A. The figure shows a representative plots of ST1, ST1/C and AT1 cell cultures. In histograms, the curves of surface stem cells markers (in red) are overlayed with the unmarked cells (in blue). B. The cumulative data of stem cell markers are represented with the floating bars; the values are expressed as median \pm Interquartile Ranges (IR) from three independent experiments (Mann-Whitney: ${ }^{*} p<0.05$ vs ST and ST/C; ${ }^{\wedge} p=$ not significant $v s \mathrm{ST}$ and ST/C). 
ST and AT cultures express stem cell-related surface markers

In order to better characterize the previously selected cultures (ST1-2, ST1-2/C and AT1-2), the expression of typical surface stem cell markers was then evaluated by flow cytometry. Because no single marker can certainly identify human tendon-derived stem or progenitor cells [18], a panel of surface antigens was examined and shown in Table 2.

FACS analysis showed pretty similar profiles of cell surface markers in cells explanted from healthy (ST) or ruptured (AT) tendons. All cultures revealed positive expression for the fibroblast marker CD90.2 (88 to $99,9 \%$ ) and for the putative mesenchymal stem markers CD44 (86,7 to 99\%) and CD105 (86,2 to 99,9\%), and were mainly negative for the hematopoietic stem cell marker CD34 (1 to 9,6\%). Nevertheless, a heterogeneous expression for the stem cell marker CD146, which is directly linked to multipotency [30], was observed. As shown in Figure 4, CD146 values were significantly higher in cells derived from healthy, ranging from 36 to $48,2 \%$ in ST1-2 and from 60,5 to $96,2 \%$ in corresponding clones ST1-2/C, compared to cells derived from ruptured samples, ranging in AT1-2 from 8,45 to 9,5\% (Figure 4A-
4B).

\section{ESWT enhances proliferation and differentiation of $S T$ and $A T$ cells}

Firstly, to evaluate the proliferative rate of our cultures in response to ESWT, we performed a Ki67 analysis -a nuclear marker of cycling cells- showing significant differences between tendon-derived cells after and before cloning, as well as from those derived from healthy ST compared to pathological AT tendons (Figure supplemental 1A). Quantitative analysis of the percentage of $\mathrm{Ki}^{+} 7^{+}$cells revealed an ESWT-mediated proliferative effect on ST1-2, ST1-2/C, and AT1-2 cultures compared with control $(p<0.05)$. Interestingly, the shock wavemediated proliferative effects were evident 12 days after the treatment -which is consistent with our previous data [11], especially in cloned (ST/C) compared to uncloned (ST and AT) cells and in ruptured (AT) versus healthy (ST) tendon-derived cells (Figure supplemental 1B).

Then the effect of ESWT on adipogenic, chondrogenic and osteogenic differentiation was evaluated using specific differentiation culture media (DM) on the previously selected 6 cultures.

Cell morphology was first assessed -by light
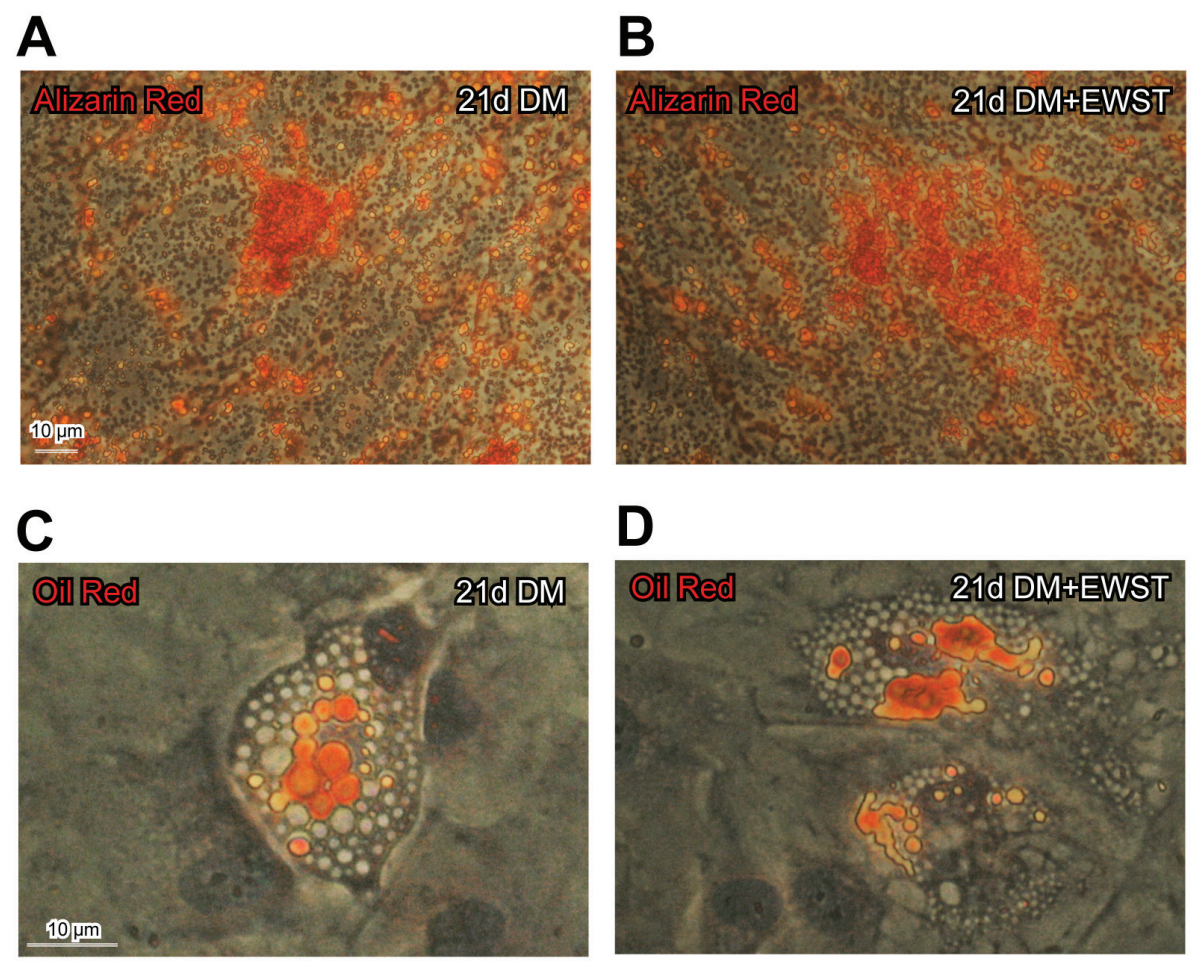

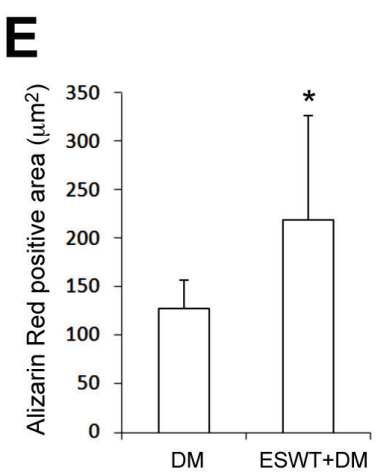

E

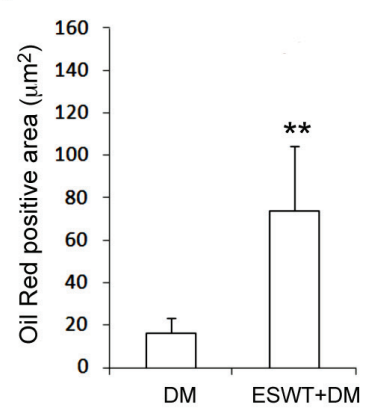

Figure 5: ESWT significantly increases the expression of differentiation markers in cells grown in specific differentiation media (DM). The clone ST1/C, representative of hTSPCs is shown for the cytochemical analysis in Figure 5A-5D (Bar: 10 $\mu$ m). Cytochemical analysis -by light microscopy- 21 days of DM cell-culture in ESWT pre-treated cells B., D. compared to DM cell culture in untreated cells A., C. Results expressed in Figure 5E-F represent the mean value \pm SD measured on 10 digital frames for each culture, for osteogenic (5A-5B, Alizarin Red depots, ${ }^{*} p<0.05 v s$ untreated) as well as adipogenic (5C-5D, Oil Red droplets, ${ }^{* *} p<0.0005 v s$ untreated) differentiation. 
Table 1: ST and AT cells share similar characteristics

\begin{tabular}{|c|c|c|c|c|}
\hline \multicolumn{5}{|l|}{ Healthy ST Cells } \\
\hline Specimen Code & Age & Fibroblast-like phenotype & Vimentin & G2/M \\
\hline ST1 & 16 & +++ & +++ & 11,4 \\
\hline ST2 & 19 & +++ & +++ & 7,5 \\
\hline ST3 & 17 & +++ & +++ & 10,5 \\
\hline ST4 & 45 & +++ & +++ & 11,2 \\
\hline ST5 & 40 & ++ & +++ & 11,1 \\
\hline Mean & 27,4 & & & 10,34 \\
\hline
\end{tabular}

\begin{tabular}{|l|l|l|l|l|}
\hline Ruptured AT Cells & \multicolumn{5}{l|}{} \\
\hline Specimen Code & Age & Fibroblast-like phenotype & Vimentin & G2/M \\
\hline AT1 & 19 & ++ & +++ & 9,1 \\
\hline AT2 & 25 & +++ & +++ & 12,1 \\
\hline AT3 & 41 & ++ & +++ & 8,7 \\
\hline AT4 & 56 & +++ & +++ & 12,5 \\
\hline AT5 & 29 & ++ & +++ & 16,9 \\
\hline Mean & $\mathbf{3 4}$ & & & $\mathbf{1 1 , 8 6}$ \\
\hline
\end{tabular}

microscopy and for few days- during the initial growth. Similarly to the results reported in Figure 1, cultured cells continued to be relatively heterogeneous, showing the same appearance described above. No significant morphological differences were observed in ESWTtreated cultures derived from healthy (ST1-2, ST1-2/C) or ruptured tendons (AT1-2) (data not shown).

The ESWT-mediated response on differentiation was further evaluated, upon 21 days in specific differentiation media (DM), by cytochemical evaluation of differentiation markers performed by the detection of Oil Red positive droplets in adipogenic medium cultured cells and Alizarin Red positive calcium-rich depots in osteogenic medium cultured cells. We never detected neither Oil Red positive droplets nor Alizarin Red calcium depots in cells cultured in DMEM basal medium. ESWT significantly increased the differentiation of ST, ST/C or AT-derived cells, grown in specific differentiation media (DM) (Figure 5A-5F). As shown in Figure 5, ESWT improved the osteogenic (detected by Alizarin Red depots) and the adipogenic (detected by Oil Red droplets) differentiation. No significant cytochemical differences were observed in cloned ST/C compared to uncloned ST1-2 and AT1-2 cultures, as well as in ST versus AT derived cells, grown in DM either pre-treated with ESWT or untreated (data not shown).

Moreover, in order to deeper investigate the cell morphology, an ultrastructural analysis by transmission electron microscopy (TEM) was performed. The substantial heterogeneity in cell appearance previously documented by light microscopy was confirmed by TEM analysis of ST, ST/C and AT cells, before or after ESWT: in fact, while most of them showed thin plasma membrane protrusions, others were characterized by an enlarged morphology lacking cell processes. Lipid droplets were clearly detectable in adipogenic medium-cultured cells (Figure 6A), whereas calcium depots are visible in nearby osteogenic medium-cultured cells (Figure 6C). The cytoplasm of the cells cultured in chondrogenic medium was heterogeneously filled with stack of regularly-aligned cisternae of rough endoplasmic reticulum and prominent Golgi apparatus, indicating their secretory activity (Figure 6B).

According to the previous analysis, again no significant morphological differences were observed in ST, ST/C or AT cultures, either before or after ESWT. In addition, TEM confirmed that ESWT enhances the in vitro-induced cell differentiation, either to adipogenic or osteogenic lineage (data not shown).

The effects of ESWT on differentiation were then assessed by RT-PCR -on the 6 cultures as above (ST12, ST1-2/C and AT1-2)- to determine the expression of specific genes activated in response to differentiative stimuli. COL2A and SOX9 expression was selected to analyze the chondrogenic lineage, ALP, BGLAP and RUNX2 for the osteogenic lineage, and PPAR $\gamma$ for the adipogenic differentiation. Results showed that, after 21 days of culture, there was an ESWT-mediated significant up-regulation in the expression of COL2A and SOX9 for chondrogenic differentiation, of ALP for osteogenic 
Table 2: FACS analysis of typical surface stem cell marker expression in ST and AT cells

\begin{tabular}{|l|l|l|l|l|l|}
\hline Surface stem cell markers expression (\%) in ST and AT-derived cells \\
\hline Specimen Code & CD146 & CD44 & CD90.2 & CD105 & CD34 \\
\hline ST1 & 36 & 95,8 & 99,6 & 99,6 & 3 \\
\hline ST2 & 48,2 & 99 & 99,9 & 97,8 & 4,4 \\
\hline ST1/C & 96,2 & 93,7 & 99,9 & 99,7 & 1 \\
\hline ST2/C & 60,5 & 91,3 & 99,2 & 95,8 & 2 \\
\hline AT1 & 8,45 & 96,2 & 99,5 & 99,6 & 9,6 \\
\hline AT2 & 9,5 & 86,7 & 88 & 86,2 & 4,4 \\
\hline
\end{tabular}

and PPAR $\gamma$ for adipogenic differentiation in all types of cells $(p<0.05$ : ESWT+DM vs DM alone), confirming the cytochemical observations (Figure 7A). In contrast, the osteogenic markers BGLAP and RUNX2 were not significantly different. The gene expression profiles of cells cultured in DMEM was used as negative control. Again, no significant differences were detectable in cultures derived from healthy semitendinosus (ST and $\mathrm{ST} / \mathrm{C}$ ) or from ruptured Achilles (AT) tendon explants (data not shown).

Further molecular analysis to control ESWTmediated effects was performed in ST, ST/C or AT cultures grown 12 days in basal medium, measuring mRNA transcript levels of the early differentiation tenocyte marker Scleraxis (Scx) (Figure 7B) [31, 32]. In untreated samples, Scx expression was significantly higher in ruptured AT-derived compared to healthy STderived cells. In ESWT-treated samples, Scx levels were significantly increased in ST/C, compared to uncloned ST and AT cultures.

In this work we designated both types of cultures as human Tendon-derived Stem/Progenitor cells (hTSPCs), according with previous report [18].

\section{DISCUSSION}

In this study, we show that ESWT is able to accelerate the in vitro-induced differentiation of human primary cultured Tendon Stem/Progenitor cells. In a previous study, we demonstrated that ESWT increases the functional activities of tenocytes explanted from human tendons -including proliferation, migration and collagen synthesis- without modifying cell viability [1113]. Moreover we also showed that cultures derived from ruptured tendons were more susceptible to shock wavemediated activation, indicating a possible recruitment of undifferentiated progenitors in the site of injury $[12,13]$. Here we confirm that ESWT induces cell proliferation, although our cultures are not at the same stage of differentiation, and are characterized by a heterogeneous natural differentiation potential, probably initiated by tendon injuries $[12,13]$. Therefore, according with other reports $[14,15,33]$, the clinical benefits of ESWT can be explained by mechanical stress promoting the in vivo regenerative capacity of the tissue.

However it is widely accepted that physiological repair after tendon damage is pretty limited [34]. As recently suggested, human stem cells are considered a suitable tool for the management of human chronic tendinopathies, albeit their effective contribution to tendon regeneration in vivo is still matter of discussion $[2,5,15$, $19,24,35,36,37]$. Cultures of tendon-derived stem cells were established and several reports investigated strategies to enhance differentiation into tenocytic-like lineage $[18,38,39]$. The International Society for Cellular Therapy (ISCT) proposed the minimal criteria to define human mesenchymal stem cells (MSCs), including the capacity to be plastic-adherent, proliferate, form colonies, express typical $\mathrm{CD}$ markers and differentiate in vitro to osteoblasts, adipocytes and chondroblasts [40].

In the present work, we describe these capacities in a variable percentage of healthy semitendinosus (ST) and ruptured Achilles (AT) human tendon-derived cells that we designated as human Tendon-derived Stem/Progenitor Cells (hTSPCs), according with previous report [18]. Since we published that the in vitro biological behaviour of cells explanted from healthy compared to ruptured tendons was significantly different [12], possible variations also in establishing human tendon-derived stem cells from those sources could be expected. Although proliferative capacity and multipotency are detectable in all our cultures, the clonogenicity potential is exclusive of healthy ST-, whereas ruptured AT-derived cells grow separately and do not form colonies. Interestingly, the expression of $\alpha$-SMA and Scx tenocytic differentiation markers is significantly enhanced in uncloned AT cells, whereas the stem cell-related surface marker CD146, probably linked to multipotency [30], was significantly higher in ST cells. Therefore, although Zhang and colleagues showed a different ability to form colonies in tendon progenitor cells derived from patellar and Achilles tendons of rabbits [19], we previously published that our cell characterization showed no significant differences between Achilles and semitendinosus tendon-derived cultures [12]. For this 

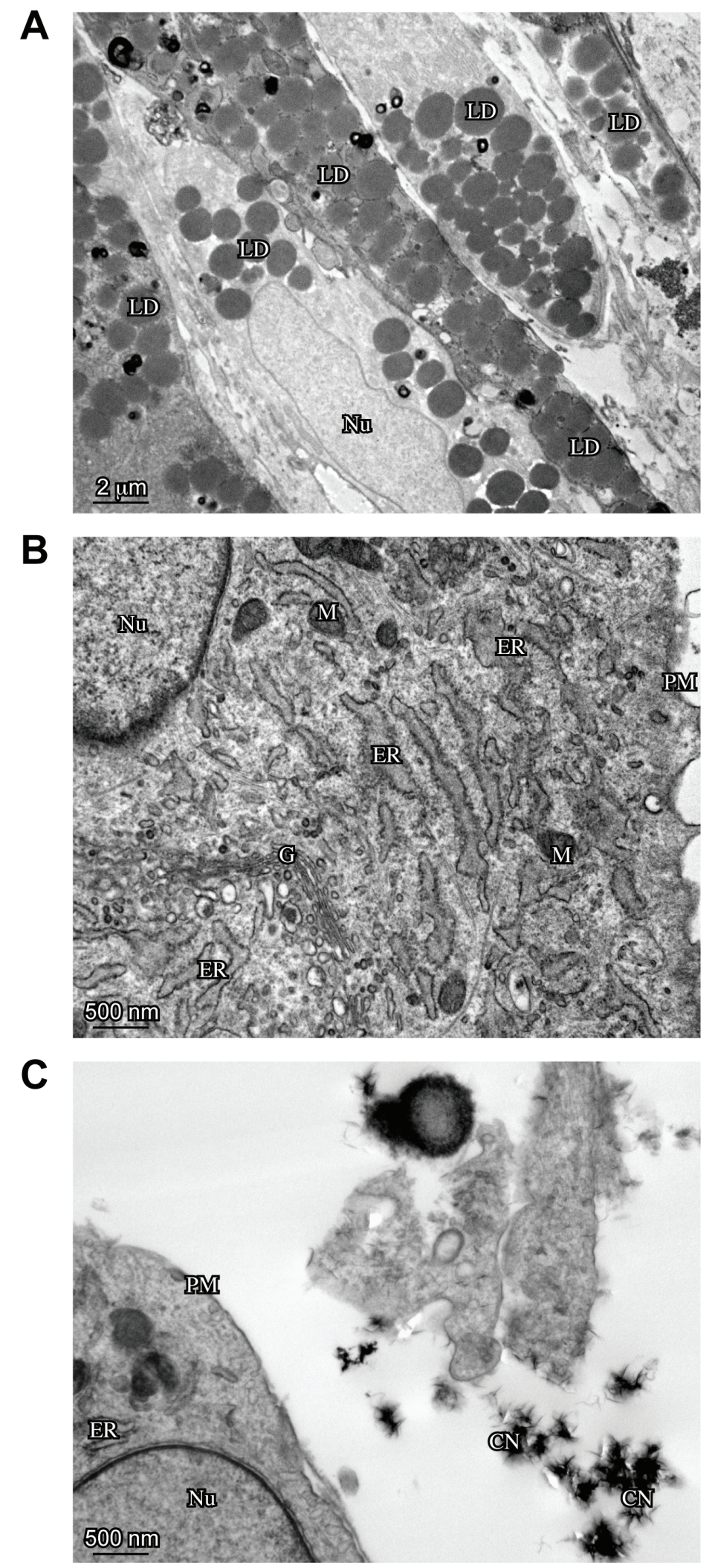

Figure 6: Ultrastructural analysis -by TEM- of ESWT-treated hTSPCs. The clone ST1/C, representative of hTSPCs is shown for the ultrastructural analysis. A. Electron microscopy analysis shows the presence of lipid droplets (LD) in adipogenic medium-cultured hTSPCs (Bar: $2 \mu \mathrm{m})$. B. hTSPCs grown in chondrogenic medium shows abundant rough Endoplasmic Reticulum (ER) and prominent Golgi apparatus (G) (Bar: 500nm). C. Calcium depots (CN: Centers of initial Nucleation) are present in osteogenic medium-cultured hTSPCs (Bar: 500nm). Mitochondrion (M), Nucleus (N), Plasma Membrane (PM). 

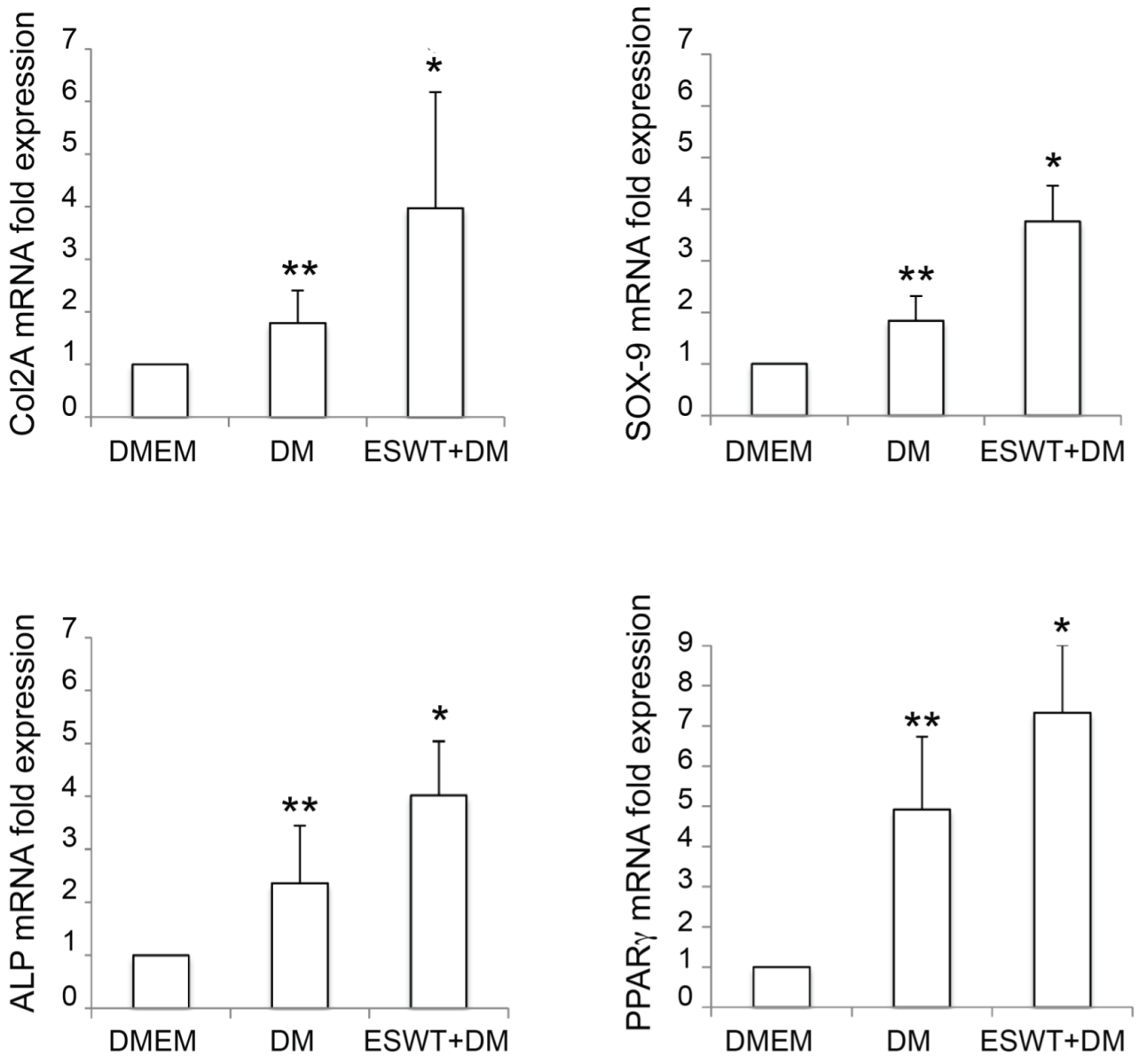

B


Figure 7: Molecular analysis of typical differentiation markers -by RT-PCR- of ESWT-treated hTSPCs. A. mRNA expression of COL2A, SOX9 (chondrogenic differentiation), ALP (osteogenic differentiation), and PPAR $\gamma$ (adipogenic differentiation) was evaluated after 21 days of culture in differentiation media (DM) following or not ESWT in ST/C, ST and AT cells; $\left({ }^{*} p<0.01\right.$ : ESWT + DM vs DM; ${ }^{* *} p<0.05$ : DM vs DMEM). Corresponding gene expression in cells cultured in DMEM was used as negative control and values were normalized to DMEM, set to 1. B. mRNA expression of Scx (early differentiation marker) was evaluated after 12 days of culture in DMEM basal medium \pm ESWT. In untreated samples, values were normalized to ST/C cells, set to $1\left({ }^{*} p<0.005\right.$ : AT $v S \mathrm{ST}$ and ST/C; ${ }^{\wedge} p=$ not significant: ST $v$ ST/C). In ESWT-treated samples, values were normalized to ST cells, set to $1\left({ }^{*} p<0.0005\right.$ : ST/C $v S$ AT and ST; ${ }^{\wedge} p=$ not significant: $\mathrm{AT} v s \mathrm{ST}$ ). 
reason, we propose that the differences observed in our samples may be amenable to the damage occurred in human AT and its physiological attempt to repair the injury, although we cannot definitively exclude that they are due to their different origin.

It is likely that a variable amount of resident hTSPCs started a differentiative program to regenerate the tissue. Intriguingly, even though uncloned ruptured AT cells already started a differentiation program toward the tenocytic lineage, all hTSPCs cultures partially maintain a multidifferentiative potential, as ascertained by their capacity to differentiate in vitro. Although the physiological repair of tendons remains difficult, it is conceivable that our tendon-derived cells can be facilitated by specific treatments, such as ESWT, to differentiate toward tenocytic-like lineage.

Consistent with this hypothesis, we show for the first time that ESWT significantly enhances hTSPCsinduced differentiation, as indicated by cytochemical and molecular findings. Our results are mainly in agreement with previous reports performed in vitro on equine adipose tissue-derived stem cells [41], rat and human adipose-derived stem cells [17, 42] and human bone marrow stromal cells $[15,16]$. Surprisingly, no significant differences in the appearance and behavior of healthy ST and ST/C compared to AT cells was induced by ESWT, implying that the different amount of hTSPCs present in both types of explants share similar characteristics. Therefore we suppose that in our cultures may coexist cellular elements bearing different degrees of differentiation, all being sensitive to shock wave stimulation.

Taken together, previous reports and the present observations seem to corroborate the long-held idea that ESWT may enhance recruitment, proliferation and differentiation of tissue-derived stem cells $[15,17,41]$. We propose that such treatment, applied on injured tendons, is able to accelerate stem cell differentiation -probably through the release of different cytokines and growth factors- mainly towards tenocytic lineage, contributing to tendon repair.

Further investigations will certainly help to better clarify that ESWT clinical benefits are amenable to mechanisms triggering tendon healing promoted by resident stem cells recruitment.

\section{MATERIALS AND METHODS}

\section{Patients}

Adult human tendon-derived cells were explanted from 5 healthy semitendinosus (ST) tendons of patients affected by Anterior Cruciate Ligament (ACL) rupture and subjected to ligament reconstruction by ST, and 5 from patients affected by Achilles (AT) tendon ruptures. The average age of the patients was $31 \pm 15$ years.

The Institutional Review Board of "Sapienza" University and Sant'Andrea Hospital approved the study protocol, and all patients gave their written informed consent to the experimental study.

\section{Isolation, culture and cloning of tendon-derived cells}

Freshly isolated tendon-derived cells, from ST or AT, were seeded in 24well/plates (or T25 plates) and cultured around 3 weeks in conventional medium (DMEM with 10\% FBS), as described [11-13]. In order to form colonies, cells were cultured 8-10 days in Tissue Culture Dishes (Corning 100x20mm) after a diluting suspension to 1 cell $/ \mu 1$, as previously described $[18,19]$. Single cell colonies, when clearly detectable and more than $2 \mathrm{~mm}$ in diameter, were detached by local application of trypsin, transferred to T25 flasks for further culture, rapidly proliferated reaching around $80 \%$ confluence and finally used for the following experiments. Also cells that were not able to form colonies were similarly detached and cultured, and used for the following experiments.

\section{Immunofluorescence}

Cells grown on coverslips were fixed with $4 \%$ paraformaldehyde followed by treatment with $0.1 \mathrm{M}$ glycine for $20 \mathrm{~min}$ at $25^{\circ} \mathrm{C}$ and with $0.1 \%$ Triton $\mathrm{X}-100$ for additional $5 \mathrm{~min}$ at $25^{\circ} \mathrm{C}$ to allow permeabilization.

Cells were then incubated alternatively with the following primary antibodies: the anti-vimentin (1:50 in PBS; Dako, Glostrup, Denmark) and alpha-smooth muscle actin ( $\alpha$-SMA) (1:50 in PBS; Dako) monoclonal antibodies and the rabbit polyclonal antibody anti-Ki67 (1:50; Zymed Laboratories; San Francisco, CA, USA). The primary antibodies were visualized, after appropriate washing with PBS, by using goat anti-mouse IgG-FITC (1:50 in PBS; Cappel Research Products, Durham, NC, USA), for $30 \mathrm{~min}$ at $25^{\circ} \mathrm{C}$ and the goat anti-rabbit IgGTexas Red (1:200; Jackson Immunoresearch Laboratories, West Grove, PA, USA). Nuclei were stained with 4'-6'-diamido-2-phenylindole dihydrochloride (DAPI) (1:10,000 in PBS, Sigma Chemical Co., St. Louis, MO, USA). Coverslips were finally mounted with Mowiol in PBS for observation. Fluorescence signals were analyzed by conventional fluorescence or by scanning cells in series of $0.5 \mu \mathrm{m}$ sequential sections with an ApoTome System (Zeiss, Oberkochen, Germany) connected with an Axiovert 200 inverted microscope (Zeiss); image analysis was then performed by the Axiovision software (Zeiss). The percentage of vimentin, $\alpha$-SMA and Ki67-positive cells was analyzed counting for each treatment a total of 500 cells, observed in ten microscopic fields randomly 
taken from three different experiments. Results have been expressed as mean values \pm Standard Deviation (SD).

\section{Flow cytometry analysis}

For the analysis of cell cycle, each culture was plated sparsely at P1 so that it did not touch each other and did not reach contact inhibition. Cells were trypsinized, pelleted and resuspended in 70\% ethanol in PBS, and stored at $4^{\circ} \mathrm{C}$ overnight. Then they were washed with PBS, resuspended in propidium iodide (PI) staining solution (50 $\mathrm{mg} / \mathrm{ml}$ ) and RNAse A (100 Kunitz/ml) (Miltenyi Biotec $\mathrm{GmbH}$, Bergisch Gladbach, Germany) and incubated in the dark for 40 minutes at room temperature.

For the surface antigen expression, the MoAbs used were: CD146-PE, human (Miltenyi Biotec $\mathrm{GmbH}$ ), CD90.2-APC (Miltenyi Biotec GmbH), CD44-FITC (Becton, Dickinson and Company, Franklin Lakes, New Jersey), CD105-APC (Miltenyi Biotec $\mathrm{GmbH}$ ) and CD34-PE (Miltenyi Biotec GmbH). Negative control was performed using the $\mathrm{HaCaT}$, spontaneously immortalized human keratinocyte cell line.

For this assay the cells were harvested and stained according to the manufacturer instructions. Each measurement contained at leats 30,000 cells collected with MACSQuant ${ }^{\circledR}$ Analyzer flow cytometer (Miltenyi Biotec $\mathrm{GmbH}$ ) and analysed with MACSQuantify ${ }^{\circledR}$ software (Miltenyi Biotec $\mathrm{GmbH}$ ).

\section{In vitro differentiation and proliferation of tendon-derived cells}

Cellular samples were then passed and further cultured in specific differentiation media for osteocytes, chondrocytes or adipocytes. Differentiation of each sample was performed using specific media to promote osteogenic, chondrogenic and adipogenic differentiation, respectively. Cells were cultured for osteogenic differentiation in hMSC Osteogenic Diff BulletKit (LONZA, Basel Switzerland), for chondrogenic differentiation in hMSC Chondrogenic Diff BulletKit and TGF- $\beta 3$ for hMSC Chondro (LONZA, Basel Switzerland), and for adipogenic differentiation in hMSC Adipogenic Diff BulletKit (LONZA, Basel Switzerland), as previously described [43].

In order to evaluate their possible influence on in vitro cell differentiation, cultures were subjected to ESWT as recently described [11-13], using an electromagnetic shock wave generator MODULITH ${ }^{\circledR}$ SLK (STORZ MEDICAL AG; Tägerwilen, Switzerland), at a dose of shock waves $0.14 \mathrm{~mJ} / \mathrm{mm}^{2}$ energy level and 1000 impulses as previously described [11-13], whereas the control group was maintained in the same culture conditions, consisting in conventional or differentiation media, without previous shock wave exposure. Shock wave intensity and energy levels were previously selected [11-13] in order to maximize the therapeutical effects without significantly decrease the cell viability.

A cryogenic vial (Corning Incorporated, NY, USA) containing a cell suspension with $1.10^{6}$ cells $/ \mathrm{ml}$ was placed on shock wave generator with a coupling gel (Aquasonic 100; Parker Laboratories, Fairfield, New Jersey, USA) to minimize the loss of shock wave energy at the interface between the head of the device and the cryovial. The tube was placed exactly in the focus of the application pad under ultrasonographic control.

In particular, cells destined to morphological/ cytochemical and molecular analysis were grown 2-3 days in the Differentiation Media (DM), detached and exposed to ESWT, and further cultured 3 weeks in DM. Controls were grown 3 weeks in conventional or differentiation media, without shock wave exposure. According with our previous reports [11, 12], cells evaluated for Ki67 proliferation and Scx early differentiation marker were grown 12 days after ESWT in conventional medium, whereas controls were grown the same days without previous shock wave exposure.

\section{Morphological analysis}

The morphological analysis of human tendonderived cells -either from healthy ST or ruptured AT- was performed during cell growth in specific differentiation media, following ESWT, using an Axiovert 200 inverted microscope (Zeiss, Oberkochen, Germany) equipped with Axiovision image analysis system (Zeiss).

\section{Transmission electron microscopy}

Human primary cultures, isolated and treated as above, were fixed with $2 \%$ glutaraldehyde in PBS at 4-C. Samples were postfixed in $1 \%$ osmium tetroxide in veronal acetate buffer ( $\mathrm{pH} \mathrm{7.4)}$ for 1 hour at $25^{\circ} \mathrm{C}$, stained with uranyl acetate $(5 \mathrm{mg} / \mathrm{ml})$ for 1 hour at $25^{\circ} \mathrm{C}$, dehydrated in acetone and embedded in Epon 812 (EMbed 812, Electron Microscopy Science, Hatfield, PA, USA). Ultrathin sections, unstained or poststained with uranyl acetate and lead hydroxide, were examined under a Morgagni 268D transmission electron microscope (FEI, Hillsboro, OR, USA), equipped with a Mega View II charge-coupled device camera (SIS, Soft Imaging System GmbH, Munster, Germany) and analyzed with AnalySIS software (SIS, Soft Imaging System GmbH, Munster, Germany).

\section{Quantitative evaluation of osteogenic and adipogenic differentiation markers}

The evaluation of ESWT potential to influence differentiation of tendon-derive cells grown in specific 
media (as described earlier) was performed by cytochemical staining of typical differentiation markers, Oil Red for adipocytes and Alizarin Red for osteocytes. In particular, after 3 weeks of culture, ESWT treated/ untreated cells were stained using Oil Red $\mathrm{O}$ assay kit (Millipore), as indicator of intracellular lipid droplets in adipocytes or Alizarin Red S assay kit (Millipore) as indicator of mineral depots in osteocytes, following manufacturer's instructions. Digital images were collected by a Axiocam (Zeiss) CCD camera and analyzed by the Axiovision (Zeiss) software. Background and positive levels of staining were defined in control slides. For Oil Red and Alizarin Red, samples -observed at a final magnification of $320 \mathrm{X}$ - were scanned for the whole length and at least 10 digital frames were randomly taken for each conditions. Values are expressed as percentage of positive area/total frame area \pm Standard Deviation (SD).

\section{Molecular analysis of chondrogenic, osteogenic, adipogenic and tenocytic differentiation}

The ESWT potential to influence differentiation of tendon-derived cells grown in osteogenic, chondrogenic and adipogenic media was further tested, determining by qRT-PCR the expression of specific genes activated in response to differentiative stimuli. Oligonucleotide primers for target genes and for the housekeeping gene were chosen with the assistance of the Oligo 5.0 computer program (National Biosciences, Plymouth, $\mathrm{MN}$ ) and purchased from Invitrogen. The following primers were used: for alkaline phosphatase, ALP target gene: 5'-CAAGGACGCTGGGAAATCTGT-3' (sense), 5'-AGGGGGCATCTCGTTGTCT-3' (anti-sense); for Bone Gamma-Carboxyglutamate (Gla) Protein, BGLAP target gene: 5'-CCTCACACTCCTCGCCCTAT-3' (sense), 5'- GCTTGGACACAAAGGCTGC-3' (antisense); for the Run-related transcription factor 2, RUNX2 target gene: 5'- GAACCCAGAAGGCACAGACA-3' (sense), 5'-GCGGGACACCTACTCTCATAC-3' (anti-sense); for the transcription factor, SOX9 target gene: 5'-CCCCCAACGCCATCTTCAA-3' (sense), 5'-CTGGGATTGCCCCGAGTG-3' (antisense); for the type II collagen, COL2A target gene: 5'- ACACTGGGACTGTCCTCTGCGA -3' (sense), 5'-CCTTTGGTCCTGGTTGCCCACTG-3' (anti-sense); for the peroxisome proliferator activator receptor gamma, PPAR $\gamma$ target gene: 5'-GGAAAGACAACAGACAAATCACCAT-3' (sense), 5'-ACCTCTTTGCTCTGCTCCTG-3' (antisense); for the transcription factor scleraxis A, SCXA target gene: 5'-CGAGCGAGACCGCACCAACA-3' (sense), 5'-CGTTGCCCAGGTGCGAGATGTAG-3' (anti-sense); for the 18S rRNA housekeeping gene: 5'-AACCAACCCGGTCAGCCCCT-3' (sense), 5'-TTCGAATGGGTCGTCGCCGC-3' (antisense). For each primer pair, we performed no-template control and noreverse- transcriptase control (RT negative) assays, which produced negligible signals. RNA was extracted using the TRIzol method (Invitrogen, Carlsbad, CA) according to manufacturer's instructions and eluted with $0.1 \%$ diethylpyrocarbonate (DEPC)-treated water. Total RNA concentration was quantitated by spectrophotometry and the quality was assessed by measuring the optical density ratio at $260 / 280 \mathrm{~nm}$. RNA samples were stored at $-80^{\circ} \mathrm{C}$. After denaturation in DEPC-treated water at $70^{\circ} \mathrm{C}$ for $10 \mathrm{~min}, 1 \mu \mathrm{g}$ of total RNA was used to reverse transcription using iScriptTM cDNA synthesis kit (BioRad) according to manufacturer's instructions. Realtime PCR was performed using the iCycler Real-Time Detection System (iQ5 Bio-Rad) with optimized PCR conditions. The reaction was carried out in 96-well plate using iQ SYBR Green Supermix (Bio-Rad) adding forward and reverse primers for each gene and $1 \mu \mathrm{l}$ of diluted template cDNA to a final reaction volume of $15 \mu \mathrm{l}$. All assays included a negative control and were replicated three times. The thermal cycling programme was performed as follows: an initial denaturation step at $95^{\circ} \mathrm{C}$ for $3 \mathrm{~min}$, followed by 45 cycles at $95^{\circ} \mathrm{C}$ for $10 \mathrm{sec}$. and $60^{\circ} \mathrm{C}$ for $30 \mathrm{sec}$. Real-time quantitation was performed with the help of the iCycler IQ optical system software version 3.0 a (Bio-Rad), according to the manufacturer's manual. The relative expression of the housekeeping gene was used for standardizing the reaction. The comparative threshold cycle $(\mathrm{Ct})$ method was applied to calculate the fold changes of expression compared to control cells. Results are reported as mean \pm SD from three different experiments in triplicate.

\section{Statistical analysis}

To compare variables that assume normal distribution, Student's T test was calculated. The values are expressed as mean $\pm \mathrm{SD}$ or $\mathrm{SE}$ from three independent experiments.

To compare variables that do not assume Gaussian distribution, Mann-Whitney non-parametric test was used. The values are expressed as median \pm Interquartile Ranges (IR) from three independent experiments. The data are represented with the floating bars or Tukey box-andwhisker plot; the central box represents the values from the lower to upper quartile (25th to 75 th percentile), the middle line represents the median, and the horizontal lines represent the minimum and the maximum value of observation range.

$P$ values $<0.05$ were assumed as statistically significant.

\section{ACKNOWLEDGMENTS}

We thank Professor Maurizio Alimandi, "Sapienza" 
University of Rome, for critically reviewing the manuscript.

\section{CONFLICTS OF INTEREST}

The authors declare that there is no conflict of interests.

\section{Authors' contributions}

M.C.V., A.F., M.R.T. and V.V. designed the project and supervised the study. L.L., S.R., M.V., D.R., F.M. and C.S. performed the experiments. S.R., F.M. and V.V. analyzed the data and performed statistics. S.R., D.R. and V.V. generated the figures. V.V. wrote the manuscript.

\section{REFERENCES}

1. Lui PP and Chan KM. Tendon-derived stem cells (TDSCs): from basic science to potential roles in tendon pathology and tissue engineering applications. Stem cell reviews. 2011; 7:883-897.

2. de Mos M, Koevoet WJ, Jahr H, Verstegen MM, Heijboer MP, Kops N, van Leeuwen JP, Weinans H, Verhaar JA and van Osch GJ. Intrinsic differentiation potential of adolescent human tendon tissue. 2007; 8:16.

3. Shi LL, Boykin RE, Lin A and Warner JJ. Association of suprascapular neuropathy with rotator cuff tendon tears and fatty degeneration. Journal of shoulder and elbow surgery. 2014; 23:339-346.

4. Ahmad Z, Wardale J, Brooks R, Henson F, Noorani A and Rushton N. Exploring the application of stem cells in tendon repair and regeneration. Arthroscopy. 2012; 28:1018-1029.

5. Ruzzini L, Longo UG, Rizzello G and Denaro V. Stem cells and tendinopathy: state of the art from the basic science to clinic application. Muscles, ligaments and tendons journal. 2012; 2:235-238.

6. Pietschmann MF, Frankewycz B, Schmitz P, Docheva D, Sievers B, Jansson V, Schieker M and Muller PE. Comparison of tenocytes and mesenchymal stem cells seeded on biodegradable scaffolds in a full-size tendon defect model. Journal of materials science Materials in medicine. 2013; 24:211-220.

7. Machova Urdzikova L, Sedlacek R, Suchy T, Amemori T, Ruzicka J, Lesny P, Havlas V, Sykova E and Jendelova P. Human multipotent mesenchymal stem cells improve healing after collagenase tendon injury in the rat. Biomedical engineering online. 2014; 13:42.

8. Ackermann PW, Domeij-Arverud E, Leclerc P, Amoudrouz $\mathrm{P}$ and Nader GA. Anti-inflammatory cytokine profile in early human tendon repair. Knee surgery, sports traumatology, arthroscopy. 2013; 21:1801-1806.

9. Yuan T, Zhang CQ and Wang JH. Augmenting tendon and ligament repair with platelet-rich plasma (PRP). Muscles, ligaments and tendons journal. 2013; 3:139-149.

10. Moraes VY, Lenza M, Tamaoki MJ, Faloppa F and Belloti JC. Platelet-rich therapies for musculoskeletal soft tissue injuries. The Cochrane database of systematic reviews. 2014; 4:CD010071.

11. Vetrano M, d'Alessandro F, Torrisi MR, Ferretti A, Vulpiani MC and Visco V. Extracorporeal shock wave therapy promotes cell proliferation and collagen synthesis of primary cultured human tenocytes. Knee surgery, sports traumatology, arthroscopy. 2011; 19:2159-2168.

12. Leone L, Vetrano M, Ranieri D, Raffa S, Vulpiani MC, Ferretti A, Torrisi MR and Visco V. Extracorporeal Shock Wave Treatment (ESWT) improves in vitro functional activities of ruptured human tendon-derived tenocytes. PloS one. 2012; 7:e49759.

13. Visco V, Vulpiani MC, Torrisi MR, Ferretti A, Pavan A and Vetrano M. Experimental studies on the biological effects of extracorporeal shock wave therapy on tendon models. A review of the literature. Muscles, ligaments and tendons journal. 2014; 4:357-361.

14. Chen YJ, Wurtz T, Wang CJ, Kuo YR, Yang KD, Huang HC and Wang FS. Recruitment of mesenchymal stem cells and expression of TGF-beta 1 and VEGF in the early stage of shock wave-promoted bone regeneration of segmental defect in rats. Journal of orthopaedic research. 2004; 22:526-534.

15. Suhr F, Delhasse Y, Bungartz G, Schmidt A, Pfannkuche $\mathrm{K}$ and Bloch W. Cell biological effects of mechanical stimulations generated by focused extracorporeal shock wave applications on cultured human bone marrow stromal cells. Stem cell research. 2013; 11:951-964.

16. Sun D, Junger WG, Yuan C, Zhang W, Bao Y, Qin D, Wang C, Tan L, Qi B, Zhu D, Zhang X and Yu T. Shockwaves induce osteogenic differentiation of human mesenchymal stem cells through ATP release and activation of P2X7 receptors. Stem Cells. 2013; 31:1170-1180.

17. Catalano MG, Marano F, Rinella L, de Girolamo L, Bosco O, Fortunati N, Berta L and Frairia R. Extracorporeal shockwaves (ESWs) enhance the osteogenic mediuminduced differentiation of adipose-derived stem cells into osteoblast-like cells. Journal of tissue engineering and regenerative medicine. 2014.

18. Bi Y, Ehirchiou D, Kilts TM, Inkson CA, Embree MC, Sonoyama W, Li L, Leet AI, Seo BM, Zhang L, Shi S and Young MF. Identification of tendon stem/progenitor cells and the role of the extracellular matrix in their niche. Nature medicine. 2007; 13:1219-1227.

19. Zhang J and Wang JH. Characterization of differential properties of rabbit tendon stem cells and tenocytes. BMC musculoskeletal disorders. 2010; 11:10.

20. Lui PP. Identity of tendon stem cells - how much do we know? Journal of cellular and molecular medicine. 2013; 17:55-64.

21. Longo UG, Lamberti A, Maffulli N and Denaro V. Tissue 
engineered biological augmentation for tendon healing: a systematic review. British medical bulletin. 2011; 98:31-59.

22. Ni M, Lui PP, Rui YF, Lee YW, Lee YW, Tan Q, Wong YM, Kong SK, Lau PM, Li G and Chan KM. Tendonderived stem cells (TDSCs) promote tendon repair in a rat patellar tendon window defect model. Journal of orthopaedic research. 2012; 30:613-619.

23. Shen W, Chen J, Yin Z, Chen X, Liu H, Heng BC, Chen $\mathrm{W}$ and Ouyang HW. Allogenous tendon stem/progenitor cells in silk scaffold for functional shoulder repair. Cell transplantation. 2012; 21:943-958.

24. Ramdass B and Koka PS. Ligament and tendon repair through regeneration using mesenchymal stem cells. Current stem cell research \& therapy. 2014; 10:84-88.

25. Ruzzini L, Abbruzzese F, Rainer A, Longo UG, Trombetta $\mathrm{M}$, Maffulli $\mathrm{N}$ and Denaro V. Characterization of agerelated changes of tendon stem cells from adult human tendons. Knee surgery, sports traumatology, arthroscopy. 2014; 22:2856-2866.

26. Rui YF, Lui PP, Wong YM, Tan Q and Chan KM. Altered fate of tendon-derived stem cells isolated from a failed tendon-healing animal model of tendinopathy. Stem cells and development. 2013; 22:1076-1085.

27. Zhou Z, Akinbiyi T, Xu L, Ramcharan M, Leong DJ, Ros SJ, Colvin AC, Schaffler MB, Majeska RJ, Flatow EL and Sun HB. Tendon-derived stem/progenitor cell aging: defective self-renewal and altered fate. Aging cell. 2010; 9:911-915.

28. Mazzocca AD, McCarthy MB, Chowaniec DM, Cote MP, Arciero RA and Drissi H. Rapid isolation of human stem cells (connective tissue progenitor cells) from the proximal humerus during arthroscopic rotator cuff surgery. The American journal of sports medicine. 2010; 38:1438-1447.

29. Zhang $\mathrm{J}$ and Wang $\mathrm{JH}$. Platelet-rich plasma releasate promotes differentiation of tendon stem cells into active tenocytes. The American journal of sports medicine. 2010; 38:2477-2486.

30. Russell KC, Phinney DG, Lacey MR, Barrilleaux BL, Meyertholen $\mathrm{KE}$ and O'Connor KC. In vitro high-capacity assay to quantify the clonal heterogeneity in trilineage potential of mesenchymal stem cells reveals a complex hierarchy of lineage commitment. Stem Cells. 2010; 28:788-798.

31. Cserjesi P, Brown D, Ligon KL, Lyons GE, Copeland NG, Gilbert DJ, Jenkins NA and Olson EN. Scleraxis: a basic helix-loop-helix protein that prefigures skeletal formation during mouse embryogenesis. Development. 1995; 121:1099-1110.

32. Li Y, Ramcharan M, Zhou Z, Leong DJ, Akinbiyi T, Majeska RJ and Sun HB. The Role of Scleraxis in Fate Determination of Mesenchymal Stem Cells for Tenocyte Differentiation. Scientific reports. 2015; 5:13149.

33. Lee IC, Wang JH, Lee YT and Young TH. The differentiation of mesenchymal stem cells by mechanical stress or/and co-culture system. Biochemical and biophysical research communications. 2007; 352:147-152.

34. Asai S, Otsuru S, Candela ME, Cantley L, Uchibe K, Hofmann TJ, Zhang K, Wapner KL, Soslowsky LJ, Horwitz EM and Enomoto-Iwamoto M. Tendon progenitor cells in injured tendons have strong chondrogenic potential: the CD105-negative subpopulation induces chondrogenic degeneration. Stem Cells. 2014; 32:3266-3277.

35. Uysal CA, Tobita M, Hyakusoku $\mathrm{H}$ and Mizuno $\mathrm{H}$. Adipose-derived stem cells enhance primary tendon repair: biomechanical and immunohistochemical evaluation. Journal of plastic, reconstructive \& aesthetic surgery : JPRAS. 2012; 65:1712-1719.

36. Notarnicola A and Moretti B. The biological effects of extracorporeal shock wave therapy (eswt) on tendon tissue. Muscles, ligaments and tendons journal. 2012; 2:33-37.

37. Smith RK, Werling NJ, Dakin SG, Alam R, Goodship $\mathrm{AE}$ and Dudhia J. Beneficial effects of autologous bone marrow-derived mesenchymal stem cells in naturally occurring tendinopathy. PloS one. 2013; 8:e75697.

38. Barboni B, Curini V, Russo V, Mauro A, Di Giacinto O, Marchisio M, Alfonsi M and Mattioli M. Indirect co-culture with tendons or tenocytes can program amniotic epithelial cells towards stepwise tenogenic differentiation. PloS one. 2012; 7:e30974.

39. Yin Z, Chen X, Chen JL and Ouyang HW. Stem cells for tendon tissue engineering and regeneration. Expert opinion on biological therapy. 2010; 10:689-700.

40. Dominici M, Le Blanc K, Mueller I, Slaper-Cortenbach I, Marini F, Krause D, Deans R, Keating A, Prockop D and Horwitz E. Minimal criteria for defining multipotent mesenchymal stromal cells. The International Society for Cellular Therapy position statement. Cytotherapy. 2006; 8:315-317.

41. Raabe O, Shell K, Goessl A, Crispens C, Delhasse Y, Eva A, Scheiner-Bobis G, Wenisch S and Arnhold S. Effect of extracorporeal shock wave on proliferation and differentiation of equine adipose tissue-derived mesenchymal stem cells in vitro. American journal of stem cells. 2013; 2:62-73.

42. Schuh CM, Heher P, Weihs AM, Banerjee A, Fuchs C, Gabriel C, Wolbank S, Mittermayr R, Redl H, Runzler $\mathrm{D}$ and Teuschl AH. In vitro extracorporeal shock wave treatment enhances stemness and preserves multipotency of rat and human adipose-derived stem cells. Cytotherapy. 2014; 16:1666-1678.

43. Kern S, Eichler H, Stoeve J, Kluter H and Bieback K. Comparative analysis of mesenchymal stem cells from bone marrow, umbilical cord blood, or adipose tissue. Stem Cells. 2006; 24:1294-1301. 